\title{
The Nature of a Problem, Problem Diagnosis, and Engineering Design
}

\author{
Scott A.C. Flemming and Clifton R. Johnston \\ Dalhousie University \\ scott.flemming@dal.ca || clifton.johnston@dal.ca
}

\begin{abstract}
This work has been motivated by the authors' past explorations into engineering culture and engineering design which have both exposed the importance of and lack of problem definition in the engineering design process. This paper builds on those previous works and reviews literature from the fields of cognitive psychology and artificial intelligence as well as medical science. Studies in cognitive science have identified that experts perform much better in the complex task of problem identification than novices and that scheme-based instruction can greatly ameliorate novice diagnostic performance in an ill-defined complex domain such as medical science. The authors argue that these results will transfer to the analogous ill-defined and complex domain that is engineering design. In this case, the conclusion of this work is for engineering educators to develop schemes (templates) to describe in broad strokes the "universe of problems" in their particular field to aid their students in diagnosing problems for the purposes of engineering design.
\end{abstract}

Keywords: schema theory, problem definition, problem diagnosis, problem construction, engineering design process, teaching design, goals, constraints

\section{INTRODUCTION}

The motivation for this paper came from two of the authors' previous works. In [1] the authors explored the idea of engineering culture by summarizing a wide variety of literature especially in the field of engineering studies (a branch of STS). The purpose of this work was to explore possible reasons for some behavioral issues seen in engineering undergrads and practitioners through the lens of cultural anthropology. A key issue was the lack of critical thinking required by engineering curricula. The significant and unexpected product of this work was the conclusion that bringing critical thinking into engineering did not mean adding more electives but rather bringing the practice of "problem definition" into many if not all of our technical courses - especially design courses [1]. The second work sought to find best practices in "problem definition" in the engineering design sphere. The results of this review were also surprising but this time for a different reason: the idea of defining the problem was remained virtually undiscussed in engineering design literature despite the common statements that solving the wrong problem was considered to be very costly [2]. This led the authors to explore some literature in requirements engineering (RE) and creative problem-solving. These areas of study provided processes for defining problems but did not describe the elements of a problem (i.e. the outcome of the problem-defining process).

The fields of artificial intelligence and cognitive psychology especially have spent much time on this question with fruitful results. The rest of this paper details those results: i) problem construction may be the most important cognitive activity in creative problem solving (it sets the stage), ii) problems can be described in terms of four key elements, iii) experts outperform novices in the task of problem construction, and iv) expert pattern recognition can be replicated to a significant degree in novices if they are provided with "schemes" to diagnose common problems.

\section{PROBLEM DIAGNOSIS IS KEY}

Problem diagnosis is extremely valuable but it is an often neglected part of the creative problem-solving process. There are many reasons and cognitive biases which can encourage individuals to ignore concerns and re-label them as someone else's problem, not problems at all [3], or simply as complaints [4]. Many cognitive biases tend to steer persons away from digging into situations and finding problems where they were not seen before in favor of business as usual [3].

Baises or no, real problems should be defined and made clear so that a solution can at least be attempted. Our profession demands that we seek to do work that is for the betterment of society and the environment even if inconvenient [5] [6]. In can also be said, somewhat crassly, that solving real ("workplace") problems is what engineers are paid to do [7], so they have a responsibility to do so and educators a responsibility to prepare them. Einstein writes on the matter, "The formulation of a problem is often more essential than its solution, which may be merely a matter of mathematical or experimental skill. To raise new questions, new 
possibilities, to regard old problems from a new angle, requires creative imagination and marks real advance in science." (as quoted in [8]). Engineers must do the hard work of defining the problem very well to make real gains and advancements for the "wicked" problems they often face.

There is evidence to suggest, however, that we as engineering educators are not enabling students to construct real world problems [9]. Engineering students graduate with the feeling that they don't know how to "do" engineering [10]; after many years of solving textbook problems students are lost and even resist complying when finally given a design task [11]. They have been habituated to the process that years of textbook exercises have taught them to expect: "Given, Find, Equations, Diagram, Solution" [12]. Although this training for automaticity in skill execution is an aspect of expert behavior we would like our students to exhibit, it is not enough [13]. The authors argue that to complete design tasks well they must also understand their problems conceptually and execute effective domain-specific strategies [13] [14] [15]. A word of caution comes from Downey who suggests that if we do not soon begin to teach our students to think critically about the goals and stakeholders of their work, the freedom to do so will be taken away from our profession and engineering will be reduced to something like IT where problems will be defined for us in "exam-paper precision" [16] [17]. Engineering educators must develop methods of problem diagnosis that engage students in critical thinking in order to avoid such a future.

In contrast, studies have shown that persons who invest significant time in problem construction tend to excel in their careers, be better recognized, and produce better results in a wide array of areas such as art, science, and political science [18]. A metanalysis suggests that problem construction is the most valuable of all cognitive processes that have to do with creativity [19]. It would not be a stretch to claim this also for creative problem solving; several authors suggest that there is no creativity without problem solving [8] [20]. ${ }^{1}$ Although engineering educators face an uphill battle against the hidden nature of problem definition, human biases, inertia, and student resistance we must give students tools to define their problems well so they can solve the right ones.

\subsection{Constructing Problems is Key in Ill- defined Contexts}

Often educators use the word problem to describe simple textbook exercises as well as more complex design problems. These two scenarios are described as "well-defined" and "ill-defined" problems in the literature. At the risk of stating the obvious, problem definition is most important in ill-defined contexts. We will now describe in more detail the difference between the two and the continuum between these two poles.

Well-defined problems are those in which the need or issue is easy to identify [4]. That is to say the goals, constraints, specifications, and actions required to move to the goal state in a context are so easily described [22] [23] that a solution is practically executed before a second thought is given to the problem [18]. Current, desired, and intermediate states can be reasonably easily represented, and the quality of those states can be practically measured [22]. Ill-defined (or "wicked," "unstructured," "workplace," or "strategic") problems are sometimes called "messes" since they are the opposite of well-defined problems [24]. Stakeholder/client goals are often mistaken, vague, incomplete, lack clarity [4]; supporting information provided or found regarding the system of interest is confusing [24]. Even if there are few or even just one stakeholder "messy" problems have conflicting values and goals [24].

Various researchers describe a problem continuum: Basadur reports that problems can be defined as illstructured, semi-structured, or well-structured [3]. Mumford cites a study that defined these three categories more precisely as (1) wholly presented (typical textbook-type questions), (2) questions where all information is provided but requires some work in "discovering" the proper elements in the information provided, and (3) problems that need to be structured by the problem solver [20]. Getzels and Csikszentmihalyi suggest the spectrum is book-ended by presented versus discovered problems [8].

As one reads an overview of the sorts of problems that engineers encounter the question becomes how one goes about structuring ill-structured problems so that they become more like well-structured problems. This is the express purpose of problem construction. "Problem construction is concerned with defining the goals, objectives, and parameters of a problem-solving effort" [20]. The purpose of this activity is to transform vague situations into better specified problem models [23]. In other words, problem construction "defines the context" within which the problem-solving effort will be

may be a key method of ameliorating this issue [43]. This will be the topic of a future paper.
1 The importance of this fact cannot be understated. Engineering has been said to lack instruction in creativity and a problem-finding approach to instruction 
exerted and so static system states as well as dynamic relationships amongst variables can be better predicted [20] [23]. This is the role of engineering educators teaching design: enabling students to transform an illdefined problem into a well-defined problem. Problems comprise several elements and if we can define these, we can define the problem.

\section{THE NATURE OF A PROBLEM}

Various authors over the years have described the elements of a problem. Jonassen claims that a problem is simply an unknown entity that has some cultural value [25]. He suggests (at a rather high-level) problem elements are structuredness (ill- or well-structured), complexity, and domain-specificity [25]. Newell and Simon focused on well-defined problems and suggested that problem elements depend on the task instructions (especially with novices) and previous experience with similar or identical problems where "programs" from long-term memory are recalled in order to produce a solution [26]. The same authors describe a problem as initial state(s) of knowledge about a particular task, goal state(s) of knowledge about the task, and operators (information processes) which transform the states [27]; others have followed suit with very similar definitions [4] in stating that a problem is some sort of need that can be described in terms of current state and end goal and possible transition states. Still others propose that a problem should be described in terms of constraints, available resources, transformations, and processes [24]. The somewhat vague term of operations can be translated as "solution plans and procedures" [4] [26]. Over the years then there has been a wide variety of suggestions as to how to really define a problem.

Of all the literature surveyed, Holyoak has the most precise yet also exhaustive definition of a problem [23]. He writes that focus need only be upon "causally relevant" elements in a context in order to define a problem well. System models should only include elements which affect the structure of the system and not simply surface characteristics [23]. He writes that an "ideal problem model is one that describes all the elements of the world that are necessary and sufficient for concrete realization of a successful solution plan." This identification of key problem elements has not been modified in the three decades since it was introduced into problem finding literature [18] [19]. The specific elements and their descriptions are:

- Goals (the desired state(s) at some level or various levels of abstraction)

- Constraints (one could consider resources to be constraints as well, as in limits to resources)

- Variables and information (note that variables could be "soft" such as stakeholders. "Information" seems to refer to data required for accurate and reasonable assignments of values to said variables).

- Actions and operations intended for transforming system state (sometimes also referred to as strategies [13], procedures [26], or trajectories [28])

Therefore, as engineers approach an ill-defined problem, they should seek to define: the system goals, constraints, variables and the information needed to approximate their values, and state transformation strategies. Developing these elements expose system structure. We argue that the exposing of said structure will cue engineers to similar problems encountered in the past and thus afford help in deciding next steps in the engineering design process (at least for experts).

\section{EXPERT PERFORMANCE IN CONSTRUCTING PROBLEMS AND IMPLICATIONS FOR TEACHING NOVICES}

Experts outperform than novices in diagnosing problems due to expert schemata. Schemata are organized and diverse cognitive structures which allow an individual to more easily recognize and identify relevant problems especially in complex, novel, or poorly-defined contexts [20] [23]. They can better identify the important causal features of a situation which help categorize the type of problem as well as the features that are critical in order to achieve the solution [23]. In contrast, novices typically focus on the wrong problem elements which are only "surface" or of little consequence with regards to attaining the goal state [23]. "Schemes" are explicit conceptual problem representations based on experts' implicit mental schemata [13] [30] [31] [32]. Schemes help simulate chunking behavior in novices [13] [30]. Very little written about schema-based instruction in the engineering realm (engineering sciences or design) but interventions have been successful in physics, math, and other sciences [9] [32] [33]. The authors expect schemebased instruction to garner positive results in engineering design.

Engineering design is not simply another scientific context to apply scheme-based instruction; the studies mentioned above have been in the context of welldefined mathematical or scientific exercises. Since we are interested in effective instructional techniques in design the most helpful literature out there may be (somewhat surprisingly) from the medical domain. The authors argue that diagnosing the correct disease in medical science is quite like problem definition in engineering design. Like medicine, engineering design seeks to identify and solve the most problematic issues 
in complex real-world systems and the concomitant conflicting goals, data gathering and quality issues, and management of multiple stakeholders.

A key assumption unearthed by medical education research was that early medical educators believed that the practice of the scientific method was essentially the same as the practice of clinical diagnosis [36]. It was assumed that the hypothetico-deductive (i.e. guess-andtest) scientific method used to generate medical knowledge was also the best method to utilize in diagnosing disease in patients [30]. However, when told to use a hypothetico-deductive approach novices and experts alike performed very poorly in a diagnostic task. Conversely, when experts used "pattern recognition" (a function of experience so this method was not available to novices) they were ten times more likely to diagnose an illness correctly [30]. When asked to use a schemebased approach, both novices and experts were five times more likely to provide a correct diagnosis [30]. In related research Beck noted that how educational materials were organized for the purposes of teaching made a significant difference in the ability of students to diagnose illnesses in patients [37]. When information was presented and categorized with respect to pathophysiology (aspects of cyanotic and acyanotic heart disease) as opposed to categorized in terms of symptom presentation (diagnostic cues) student were much less likely to diagnose typical as well as atypical cases of the diseases properly [37]. Scheme-based instruction works in ill-defined systems. Although the conclusion sounds somewhat obvious (of course if one teaches diagnosis then students will diagnose better) medical educators were not teaching diagnostic schemes to students at the time to help them diagnose well. We argue, that right now, the same could be said about engineering educators: we are not presenting our teaching materials to facilitate design; rather we are teaching students encyclopedic knowledge in engineering science [10] [11] [12] [16] [34]. Schemebased instruction will work in the ill-defined context that is engineering design.

\section{DEFINING THE UNIVERSE: CREATING SCHEMES FOR ENGINEERING DESIGN}

In the preceding pages the authors have argued for the need of scheme-based instruction in engineering design. If this premise is accepted, the next step is to begin to create a "universe" of schemes for our students [32]. Once Mandin saw the strength of the effect of organizing teaching material in terms of diagnostics instead of pathophysiology he called for a "new taxonomy of medical problems" so that the "comprehensive knowledge domain" could be more appropriately structured for knowledge acquisition and usage [36]. The authors agree that in our profession of similarly wide fields a new taxonomy should also be developed. Such a reorganization will allow for the "systematic screening" of problem representations that Mumford et al. refer to in their desire to find the best and most creative solutions to real problems [20]. In the case of engineering design, the schemes presented are intended to help students recognize problems in terms of their causally relevant structures. Our schemes will not cue students to key medical symptoms but rather key goals, constraints, variables, and strategies for particularly important and common problems in our domains [14] [15]. The next step, then, is for engineering educators to create schemes for their particular area to help their student engineering designers recognize the symptoms of an "ill" system and define the problem in terms of goals, constraints, variables, and strategies. The authors have chosen to do just this in the discipline of Industrial Engineering (IE) and are in the process of developing categories from the past several years of capstone projects in IE. Future work will detail these categories and the "schemes" that will intend to aid students in identifying their design problem as belonging to one or more of these groups.

\section{SUMMARY}

Research suggests the problem definition stage of the design process has the greatest impact on creative and successful solutions. Novices can learn to more effectively define problems if they think of problems as containing the elements: goals, constraints, variables, and strategies. The medical domain has shown that novices can replicate expert behaviour in diagnosis if they are taught diagnostic schemes. The authors argue these results will carry over to the context of engineering design.

\section{Acknowledgements}

The authors would like to thank NSERC for their support of this research.

\section{REFERENCES}

[1] S. A. C. Flemming, C. R. Johnston and S. MacAulay Thompson, "Becoming aware of Engineering Culture: Toward sculpting a more human engineer," Proceedings of the 2018 Canadian Engineering Education Association (CEEA-ACEG18) Conference, 2018.

[2] S. A. C. Flemming and C. R. Johnston, "The Engineering Design Process - Diagnosing the Problem: "Take Time to Define" with Contextual Inquiry," Proceedings of CEEA 2019 - Learning 
to Learn - Preparing Tomorrow's Engineers, 2019.

[3] M. Basadur, "A New Methodology for Formulating Ill-Structured Problems," International Journal of Management Science, vol. 22, no. 6, pp. 627-645, 1994.

[4] G. A. Klein and J. Weitzenfeld, "Improvement of Skills for solving ill-defined problems," Educational Psychologist, vol. 13, no. 1-3, pp. 3131, 1978.

[5] N. S. o. P. Engineers, "Code of Ethics," 2020. [Online]. Available: https://www.nspe.org/resources/ethics/codeethics. [Accessed 3 February 2020].

[6] E. Canada, "Public Guideline on the code of Ethics," March 2016. [Online]. Available: https://engineerscanada.ca/publications/publicguideline-on-the-code-of-ethics\#-the-code-ofethics. [Accessed 3 Feb 2020].

[7] D. Jonassen, J. Strobel and C. B. Lee, "Everyday Problem Solving in Engineering: Lessons for Engineering Educators," Journal of Engineering Education, no. April, pp. 139-151, 2006.

[8] J. Getzels and M. Csikszentmihalyi, "From Problem Solving to Problem Finding," in Perspectives in Creativity, Chicago, Aldine, 1975, pp. 90-116.

[9] S. Hoover and J. Feldhusen, "Scientific Problem Solving and Problem Finding: A Theoretical Model," in Problem Finding, Problem Solving, and Creativity, Norwood, Ablex, 1994, pp. 201221.

[10] A. Hutchins, "Why don't future engineers learn real-world skills in school?," Maclean's, 26 Sept 2015.

[11] G. Downey and J. Lucena, "When students resist: Ethnography of a senior design experience in engineering education," International Journal of Engineering Education, vol. 19, no. 1, pp. 168-176, 2003.

[12] G. L. Downey, "The Engineering Cultures Syllabus as Formation Narrative: Critical Participation in Engineering Education through Problem Definition,," St. Thomas L.J. , vol. 428, 2008 .

[13] L. E. Hardin, "Problem-Solving Concepts and Theories," Journal of Veterinary Medicine, vol. 30, no. 3, 2003.

[14] L. J. Ball, T. C. Ormerod and N. J. Morley, "Spontaneous analogising in engineering design: a comparative analysis of experts and novices," Design Studies, vol. 25, pp. 495-508, 2004.
[15] J. Daugherty and N. Mentzer, "Analogical Reasoning in the Engineering Design Process and Technology Education Applications," Journal of Technology Education, vol. 19, no. 2, pp. 7-21, 2008.

[16] G. Downey, "ARE ENGINEERS LOSING CONTROL OF TECHNOLOGY? From 'Problem Solving' to 'Problem Definition and Solution' in Engineering Education," Chemical Engineering Research and Design, vol. 83, no. A6, pp. 583-595, 2005.

[17] G. L. Glegg, The Design of Design, New York: Cambridge University Press, 1969.

[18] R. Reiter-Palmon and E. Robinson, "Problem identification and construction: What do we know, what is the future?," Psychology of Aesthetics, 2009.

[19] R. Reiter-Palmon, "The Role of Problem Construction in Creative Production," The Journal of Creative Behavior, vol. 51, no. 4, p. 323-326, 2017.

[20] M. D. Mumford, R. Reiter-Palmon and M. Redmood, "Problem construction and cognition: Applying problem representations in ill defined domains," in Problem finding, problem solving, and creativity, Norwood, NJ, Ablex, 1994, pp. 339.

[21] H. A. Simon, "The Structure of Ill Structured Problems," ARTIFICIAL INTELLIGENCE, vol. 4, pp. 181-201, 1973.

[22] K. J. Holyoak, "Mental models in problem solving," in Tutorials in learning and memory, New York, Freeman, 1984, pp. 193-218.

[23] C. R. Schwenk, "LABORATORY RESEARCH ON ILL-STRUCTURED DECISION AIDS: THE CASE OF DIALECTICAL INQUIRY*," Decision Sciences, vol. 14, pp. 140-144, 1983.

[24] D. H. Jonassen, "Toward a Design Theory of Problem Solving," Educational Technology Research and Development, vol. 48, no. 4, pp. 6385, 2000.

[25] H. Simon and A. Newell, "Human problem solving: The state of the theory in 1970.," American Psychologist, vol. 26, no. 2, pp. 145159, 1971.

[26] A. Newell and H. A. Simon, Human Problem Solving, Englewood Cliffs: Prentice-Hall, 1972.

[27] J. R. Hajdukiewicz and K. J. ,. E. R. G. Vicente, "Development of an Analytical Framework and Measurement Tools to Assess Adaptive Performance of Individual and Teams in Military 
Work Domains," Cognitive Engineering Laboratory, Toronto, 1999.

[28] C. D. Wickens, J. G. Hollands, ,. S. Banbury and R. Parasuraman, Engineering Psychology and Human Performance, New York: Psychology Press, 2013.

[29] S. Coderre, H. Mandin, P. Harasym and G. Fick, "Diagnostic reasoning strategies and diagnostic success," MEDICAL EDUCATION, vol. 37, pp. 695-703, 2003.

[30] M. Gick and K. J. Holyoak, "Schema Induction and Analogical Transfer," COGNITIVE PSYCHOLOGY, vol. 15, pp. 1-38, 1983.

[31] S. P. Marshall, Schemas in Problem Solving, Cambridge: Cambridge University Press, 1995.

[32] A. Schoenfeld, "Teaching Problem-Solving Skills," American Mathematics Monthly, vol. 87, pp. 794-805, 1980.

[33] R. Streveler, T. Litzinger, R. Miller and P. Steif, "Learning Conceptual Knowledge in the Engineering Sciences: Overview and Future Research Directions," Journal of Engineering Education, vol. July, 2008.

[34] E. Redish and K. Smith, "LOOKING BEYOND CONTENT: SKILL DEVELOPMENT FOR ENGINEERS," Journal of Engineering Education, vol. 97, no. 295-307, 2008.

[35] H. Mandin, A. Jones, W. Woloschuk and P. Harasym, "Helping students learn to think like experts when solving clinical problems.,"
Academic Medicine, vol. 72, no. 3, pp. 173-179, 1997.

[36] A. Beck and D. Bergman, "Using Structured Medical Information to Improve Students' Problem-Solving Performance," Journal of Medical Education, vol. 61, pp. 749-756, 1986.

[37] G. Groen and V. Patel, "Medical problem-solving: some questionable assumptions," Medical education, vol. 19, pp. 95-100, 1985.

[38] D. Jonassen, "Using Cognitive Tools to Represent Problems," Journal of Research on Technology in Education, vol. 35, no. 3, pp. 362-381, 2003.

[39] D. H. Jonassen, Learning to Solve Problems: An instructional Design Guide, San Francisco: Pfeiffer, 2004.

[40] B. J. Kuipers, "Reasoning with qualitative models," Artificial Intelligence, vol. 59, pp. 125132, 1993.

[41] C. Burns and J. Hajdukiewicz, Ecological interface design, Boca Raton: CRC Press, 2004.

[42] K. Vicente and J. Rasmussen, "Ecological Interface Design: Theoretical Foundations," IEEE TRANSACTIONS ON SYSTEMS, MAN, AND CYBERNETICS, vol. 22, no. 4, pp. 589-606, 1992.

[43] E. May and D. Strong, "Is Engineering Education Delivering What Industry Requires?," Proceedings of the Canadian Design Engineering Network (CDEN) Conference, Toronto, Canada, July 24-26, 2006, 2006. 